\title{
Platelet-activating factor promotes motility in breast cancer cells and disrupts non-transformed breast acinar structures
}

\author{
V. LIBI ANANDI, K.A. ASHIQ* ${ }^{*}$ K. NITHEESH* and M. LAHIRI \\ Indian Institute of Science Education and Research, Pashan, Pune, Maharashtra 411008, India
}

Received July 20, 2015; Accepted September 4, 2015

DOI: $10.3892 /$ or.2015.4387

\begin{abstract}
A plethora of studies have demonstrated that chronic inflammatory microenvironment influences the genesis and progression of tumors. Such microenvironments are enriched with various lipid mediators. Platelet activating factor (PAF, 1-alkyl-2-acetyl-sn-glycero-3-phosphocholine) is one such lipid mediator that is secreted by different immune cell types during inflammation and by breast cancer cells upon stimulation with growth factors. Overexpression of PAF-receptor has also been observed in many other cancers. Here we report the possible roles of PAF in tumor initiation and progression. MCF10A, a non-transformed and non-malignant mammary epithelial cell line, when grown as 3D 'on-top' cultures form spheroids that have a distinct hollow lumen surrounded by a monolayer of epithelial cells. Exposure of these spheroids to PAF resulted in the formation of large deformed acinar structures with disrupted lumen, implying transformation. We then examined the response of transformed cells such as MDA-MB 231 to stimulation with PAF. We observed collective cell migration as well as motility at the single cell level on PAF induction, suggesting its role during metastasis. This increase in collective cell migration is mediated via PI3-kinase and/or JNK pathway and is independent of the MAP-kinase pathway. Taken together this study signifies a novel role of PAF in inducing transformation of non-tumorigenic cells and the vital role in promotion of breast cancer cell migration.
\end{abstract}

Correspondence to: Dr Mayurika Lahiri, Indian Institute of Science Education and Research, Dr Homi Bhabha Road, Pashan, Pune, Maharashtra 411008, India

E-mail: mayurika.lahiri@iiserpune.ac.in

*Contributed equally

Abbreviations: PAF, platelet-activating factor; PAF-R, platelet-activating factor receptor; 3D, three-dimensional; JNK, c-Jun N-terminal kinase; MAP, mitogen-activated kinase; PI3K, phosphoinositide 3-kinase; EGF, epidermal growth factor; EHS, Engelbreth-Holm-Swarm

Key words: platelet-activating factor, collective cell migration, single-cell motility, breast cancer, transformation, spheroids

\section{Introduction}

Microenvironment and tumor infiltrate have been shown to have a profound effect on different stages of cancer development ranging from cancer cell initiation, promotion and progression $(1,2)$. Chronic inflammatory microenvironments have been demonstrated to be a major predisposing factor for different cancers including breast and ovarian cancers (3-5). Phospholipid mediators such as prostaglandins (PG), lysophosphatidic acid (LPA) and platelet-activating factor (PAF) have been shown to play a role in a number of biological pathways including inflammatory diseases, cardiovascular homeostasis as well as in cancer (6-8). The role of PAF in various immunological responses like platelet aggregation, stimulation of neutrophils and macrophages, inflammation and allergic responses has also been demonstrated (9). PAF has been shown to induce apoptotic cell death in primary neurons of mice independent of the PAF receptors (10). PAF is one of the most potent mediators, which has been shown to play a vital role in neo-angiogenesis $(3,5,11)$. Interference with the PAF pathway in breast cancer, prostate cancer and Kaposi's sarcoma has been demonstrated to inhibit growth of tumors primarily due to inhibition of angiogenesis (12). Signaling through PAF has been suggested to play a role in murine melanoma lung metastasis $(13,14)$. Association of PAF to early events of transformation has been shown in BRCA-1 mutant ovarian cells (15).

PAF mediated activity occurs via its cell surface and intracellular $G$ protein-coupled receptors known as PAF receptors (PAF-R) $(16,17)$. Overexpression of PAF-R was shown to induce melanocytic tumorigenesis in transgenic mice (18). Also, upregulation of PAF receptors has been observed in invasive breast cancer cells $(16,17)$. Furthermore, use of PAF-R antagonist inhibited growth and differentiation of human breast cancer cells (19). In case of nude mice, treatment with PAF-R antagonist caused inhibition of xenografts of human prostatic carcinoma (20). Thus, highlighting the role of PAF-PAF-R pathway in the genesis and maintenance of the tumor.

Bussolati et al (16) reported that breast cancer cells MDA-MB 231, a metastatic cell line and MCF7, adenocarcinoma, secreted PAF under the influence of different growth factors, and that PAF increased the motility of MDA-MB 231 cells. However the role of PAF in breast cancer has not been studied extensively, particularly with respect to 
early events in breast cancer initiation. This study elucidated the role of PAF in breast cancer initiation, progression and promotion. To investigate the potential of PAF to induce early transformation, 3-dimensional cultures of non-tumorigenic mammary epithelial cells were grown on laminin-rich basement membrane and allowed to differentiate and organize to form polarized structures called spheroids. These spheroids have an outer layer of epithelial cells encircling a hollow lumen $(21,22)$. This well-architectured structure is vital for the form and function of the epithelial cells (23). The disruption of the well-formed polarized architecture is seen during early stages of breast cancer (21). Exposure of MCF10A, a non-tumorigenic mammalian breast epithelial cell to PAF resulted in the formation of abnormal acinar structures. These abnormal acini showed different phenotypes such as spheroids with multiple layers of cells surrounding the lumen or absence of a distinct lumen as well some acini had protrusion-like structures. The PAF-treated spheroids also showed an increase in size with a significant increase in the number of cells in each acini compared to the untreated acini. Collectively these phenotypes signify transformation. Thus, this study reports for the first time the potential of PAF to induce early transformation in breast epithelial cells.

Furthermore, to investigate the role of PAF in cancer promotion, MDA-MB 231 invasive breast cancer cells were treated with PAF to test for its ability to enhance migration. 'Collective cell migration' has been reported to play a vital role in invasion and metastasis (24). We studied this dynamic process using wound-healing assay as reported previously (25). Our study demonstrated the ability of PAF to induce enhanced collective cell migration and henceforth indicating its possible role in breast cancer promotion. At the single cell level, PAF induction showed increased motility of MDA-MB 231 cells when compared to un-induced cells, which was measured by taking into account motility parameters such as distance and displacement of an individual cell. Significant increase in velocity (rate of migration) of the cells was observed similarly to that demonstrated previously (16), however, there was no change in directionality between untreated and PAF-treated cells.

\section{Materials and methods}

Cells and culture conditions. MDA-MB 231 and MCF10A cell lines were generous gifts from Dr Kundan Sengupta (IISER, Pune) and Professor Raymond C. Stevens (The Scripps Research Institute, CA, USA), respectively. MDA-MB 231 cells were cultured in Dulbecco's modified Eagle's medium (DMEM), supplemented with $10 \%$ fetal bovine serum (FBS; Invitrogen), $2 \mathrm{mM}$ L-glutamine and $100 \mathrm{U} / \mathrm{ml}$ penicillinstreptomycin (Invitrogen). Cells were grown in 100-mm tissue culture treated dishes (Corning) at $37^{\circ} \mathrm{C}$ in a humidified $5 \% \mathrm{CO}_{2}$ incubator. MCF10A cells were cultured in growth medium containing high glucose DMEM without sodium pyruvate and 5\% horse serum (both from Invitrogen), $20 \mathrm{ng} / \mathrm{ml}$ EGF, $0.5 \mu \mathrm{g} / \mathrm{ml}$ hydrocortisone, $100 \mathrm{ng} / \mathrm{ml}$ cholera toxin and $10 \mu \mathrm{g} / \mathrm{ml}$ insulin (all from Sigma) and $100 \mathrm{U} / \mathrm{ml}$ penicillinstreptomycin (Invitrogen). High glucose DMEM without sodium pyruvate containing $20 \%$ horse serum and $100 \mathrm{U} / \mathrm{ml}$ penicillin-streptomycin (Invitrogen) was used for resuspension during sub-culturing.
RNA extraction and $c D N A$ synthesis for PAF receptor expression analysis. Cells were harvested from the culture dish using TRIzol (Ambion) by scraping cells using cell scraper (Corning), followed by DNAse I treatment using TURBO DNA-free ${ }^{\mathrm{TM}} \mathrm{kit}$ as per the manufactures protocol. Extracted RNA was quantified and cDNA was prepared using standard protocols with $5 \mu \mathrm{g}$ of RNA, $1 \mu \mathrm{l}$ oligodT (50 mM; Invitrogen) in nuclease free water (NFW), dNTP (2.5 mM) and MLMV-reverse transcriptase (Promega). Reverse transcriptase was heat inactivated at $67^{\circ} \mathrm{C}$ for $10 \mathrm{~min}$. The cDNA was further used to analyze the expression of PAF receptor in the cell lines. Amplification of the target was performed in thermal mastercyclers (Eppendorf) using forward, (5'-TACTGCTCTGTGGCCTTCCT-3') and reverse, (5'-CTGCCCTTCTCGTAATGCTC-3') primers. GAPDH was used as the house-keeping gene and was amplified with forward, (5'-ACCACAGTCCATGCCATCAC-3') and reverse (5'-TCCACACCCTGTTGCTGTA-3') primers. The following PCR cycle was used for the amplification $95^{\circ} \mathrm{C}$ for $60 \mathrm{sec}, 55^{\circ} \mathrm{C}$ for $45 \mathrm{sec}, 72^{\circ} \mathrm{C}$ for $60 \mathrm{sec}$ and final extension for $3 \mathrm{~min}$. The experiments were repeated three times to confirm the receptor status. The quantification (densitometry) was done using the gel analysis function of ImageJ software and normalized to the housekeeping gene (GAPDH) and further adjusted with respect to MCF10A expression (considering MCF10A PAF-R expression to be 1).

Wound-healing assay. Cells were seeded at a density of $5 \times 10^{5}$ cells $/ \mathrm{ml}$ of complete culture medium in culture plates with wound healing inserts (Ibidi GmbH, Munich, Germany), as per manufacturers protocol for 16-18 h. The monolayer was then treated with $10 \mu \mathrm{g} / \mathrm{ml}$ mitomycin $\mathrm{C}$ (Sigma) in serum free media for $2 \mathrm{~h}$ so as to inhibit cell proliferation (26). Cells were pre-treated with various inhibitors as per the experiment. WEB $2086(200 \mu \mathrm{M})$ (PAF receptor antagonist) $(27,28)$ was added $10 \mathrm{~min}$ prior to start of the experiment. UO126 $(10 \mu \mathrm{M})$, a MEK inhibitor (29), was added $30 \mathrm{~min}$ before PAF induction while wortmannin (200 nM) (30), PI3-K inhibitor and SP $600125(75 \mu \mathrm{M})$, JNK inhibitor (31) were added 60 min prior to PAF induction. Following treatment with inhibitors and before addition of PAF (200 nM), the inserts were removed carefully and cells were washed gently using $1 \mathrm{X}$ phosphatebuffered saline (PBS; PAN-Biotech $\mathrm{GmbH}$ ) to remove floating cells and cells were replenished with complete media containing inhibitors. Images of the wounds were acquired using phase contrast microscope (Nikon) at x10 magnification at different time-points (namely 0, 18 and $24 \mathrm{~h}$ ) after PAF induction. The results presented are average from three independent biological replicates. Wound areas were quantified using ImageJ and graphs were plotted using GraphPad Prism 6. The percentage of wound closure was calculated as:

$\%$ Wound closure $=\frac{(\text { Initial wound area }- \text { final wound area })}{\text { Initial wound area }} \times 100$

Single cell migration assay. Cells were seeded at a density of 5,000 cells/well in an 8-well chamber cover glass (LabTek) precoated with $10 \mu \mathrm{g} / \mathrm{ml}$ fibronectin $\left(2-3 \mathrm{~h}\right.$ treatment at $\left.37^{\circ} \mathrm{C}\right)$. Cells were maintained at $37^{\circ} \mathrm{C}$ in complete medium for $18-20 \mathrm{~h}$. For live cell imaging, cells were supplemented with L-15 medium 
containing $10 \% \mathrm{FBS}$ and $100 \mathrm{U} / \mathrm{ml}$ penicillin-streptomycin (both from Invitrogen) containing WEB 2086 and PAF as per the experiment. Time-lapse microscopy for $4 \mathrm{~h}$ was carried out in a stage incubator using Zeiss LSM 710 laser scanning confocal microscope at x10 magnification. Cells were tracked in ImageJ analysis software using manual tracking plugin. Randomly selected cells from three to five independent experiments were tracked and distance travelled, displacement and velocity of a cell were calculated using Chemotaxis and Migration tool (Ibidi GmbH).

$3 D$ 'on top' cultures and immunofluorescence analysis. MCF10A cells were grown as 3D-'on top' cultures according to standard protocols $(21,32,33)$. PAF was added on day $4,8,12$ and 16 into the culture medium (day of seeding was considered as day 0) and the acini were harvested on day 20 and immunostained as outlined earlier $(21,32,33)$ with phalloidin (Invitrogen) instead of Alexa Fluor-conjugated secondary antibody to label the actin cytoskeleton. Images were acquired using Zeiss LSM 710 laser scanning confocal microscope at x63 magnification. The results depicted are representative images from three independent biological experiments. A total of 90 acini were imaged and optical sections were analyzed and classified based on the observed phenotype. Graphs were plotted and statistical analysis was done using GraphPad Prism software (GraphPad Software, La Jolla, CA, USA). Measurement of the volume of acini and counting of number of nuclei were done using Image-Pro Plus software (Media Cybernetics, USA). Around 30 acini were analyzed each in control and treated.

Ethics. This study did not involve utilization of human subject and has been carried out using cell lines.

Statistical analysis. Wound healing data are presented as mean \pm SEM. One-way ANOVA was used to test the significance of difference of the percentage of wound closure across treatments. Mann-Whitney $U$ test was used to analyze the significance of difference between parameters used in single cell migration assay (accumulated distance, euclidean distance, velocity and directionality and 3D morphometric analysis). $\mathrm{P}<0.05$ was considered statistically significant. ${ }^{* * *}$, ** and * indicate $\mathrm{P}<0.0001, \mathrm{P}<0.01$, and $\mathrm{P}<0.05$, respectively.

\section{Results}

PAF induces formation of abnormal acinar structures in immortalized non-tumorigenic breast epithelial cell line MCF10A grown as 3D 'on-top' cultures. MCF10A cells are spontaneously immortalized, non-tumorigenic cells of normal breast epithelial origin. These cells when grown on laminin rich extracellular matrix form multicellular acini-like structures resembling epithelial cells lining the acinus, the smallest functional unit of a human mammary gland (34). MCF10A cells give rise to acinar structures wherein the hollow lumen is surrounded by a single layer of epithelial cells as shown in Fig. 1A (top panel). Upon exposure to PAF, these well-organized structures were observed to be disrupted. Sixty-five percent of the spheroids showed multiple layers of cells surrounding a lumen or absence of a distinct lumen (Fig. 1A; middle panel and B) while $\sim 58 \%$ showed formation of protrusion-like structures as seen in Fig. 1A (bottom panel) and C. Further morphometric analysis revealed that the PAF-treated acini were larger in size as indicated by a significant increase in volume compared to untreated acini (Fig. 1E). Also there was a significant increase in the number of cells per acini (Fig. 1F), which implied that PAF induces proliferation of MCF10A cells in the acini. Taken together, PAF was found to disrupt overall morphology of the spheroids as well as induce proliferation, an indicator of transformation.

PAF increases collective cell migration in MDA-MB 231. Since PAF treatment induced formation of disrupted acinar structures, we checked for the PAF-R status in MCF10A as well as in two other breast cancer cells, MDA-MB 231 and MCF7s, using RT-PCR. PAF-R expression levels in these cell lines were correlated with their tumorigenic potential. MDA-MB 231 and MCF7 cells showed overexpression of PAF-receptors while MCF10A showed weak expression (Fig. 2A and B). However there was no appreciable change in the expression levels of the receptor with increase in dose of PAF (data not shown). The effect of PAF on collective cell migration was investigated using wound healing assay. MDA-MB 231 cells were treated with different doses of PAF ranging from 10 to $200 \mathrm{nM}$. PAF (200 nM) showed an appreciable increase in motility by $18 \mathrm{~h}$ (data not shown). Thus, further experiments were performed using $200 \mathrm{nM}$ PAF. On exposure of MDA-MB 231 cells to $200 \mathrm{nM}$ PAF, the cells showed a significant increase in migration with almost $80 \%$ wound closure at $18 \mathrm{~h}$ (Fig. 2C and D). This increase in motility was inhibited upon pre-treatment of cells with WEB 2086, a PAF receptor antagonist $(27,28)$. Thus, confirming role of PAF in inducing increased migration of highly invasive MDA-MB 231 cells.

PAF-induced migration is mediated via PI3-kinase pathway and/or JNK pathway, but not via MAP-kinase pathway. To predict the possible pathway(s) involved in PAF-induced cell migration scratch assays along with inhibitors for generally known motility pathways were performed. It was observed that the MAP-kinase pathway did not mediate PAF-induced migration since inhibition of the pathway with a MEK inhibitor, UO126 (29), did not have an effect on wound closure (Fig. 3A).

PAF stimulated cells, upon pre-treatment with wortmannin, a PI3-K inhibitor (30), showed wound closure which was similar to unstimulated and untreated cells and was significantly less than PAF-treated cells (Fig. 3B). Thus, indicating a possible role of PI3-K pathway in PAF induced motility. However, the wound closure of unstimulated wortmannin-treated cells was significantly less than control cells indicating the role of PI3-kinase pathway in normal MDA-MB 231 cell motility (Fig. 3B).

Use of the JNK inhibitor SP600125 (31), also abrogated both PAF-induced as well as inherent motility of MDA-MB 231 cells (Fig. 3C). However, the extent of inhibition of migration of PAF stimulated cells as well as untreated cells by the inhibitor suggests the possible role of JNK pathway in PAF induced motility.

$P A F$ induces chemokinesis at the single cell level in MDA-MB 231 cells. To determine the effect of PAF on 
A
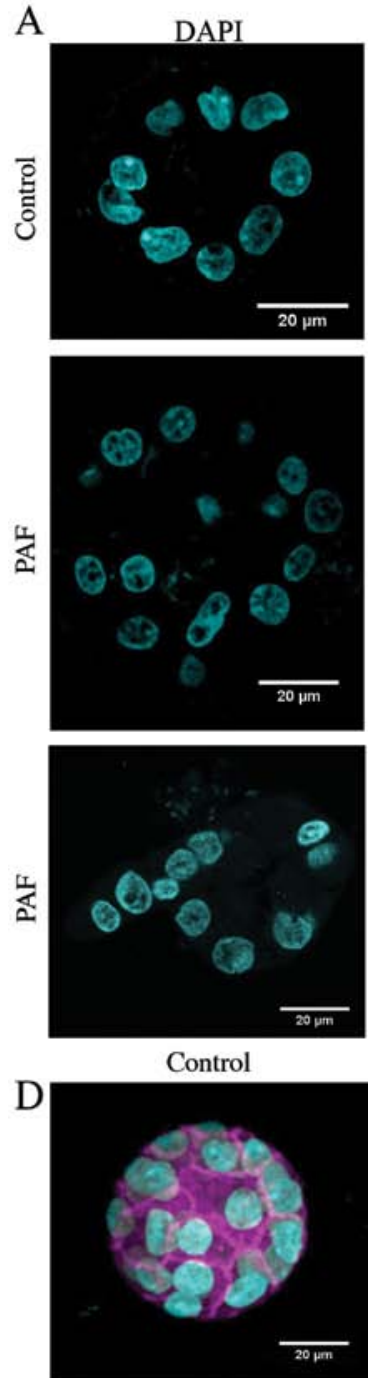

$\mathrm{E}$

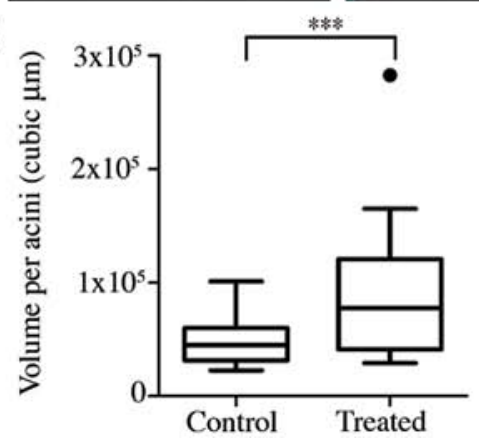

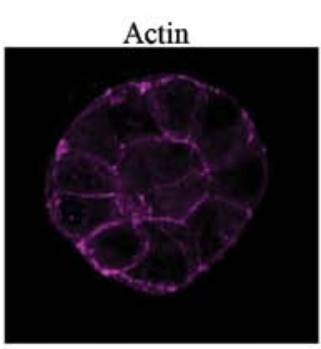
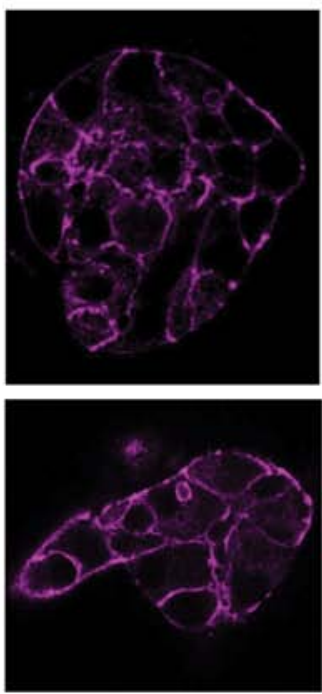

$200 \mathrm{nM}$ PAF
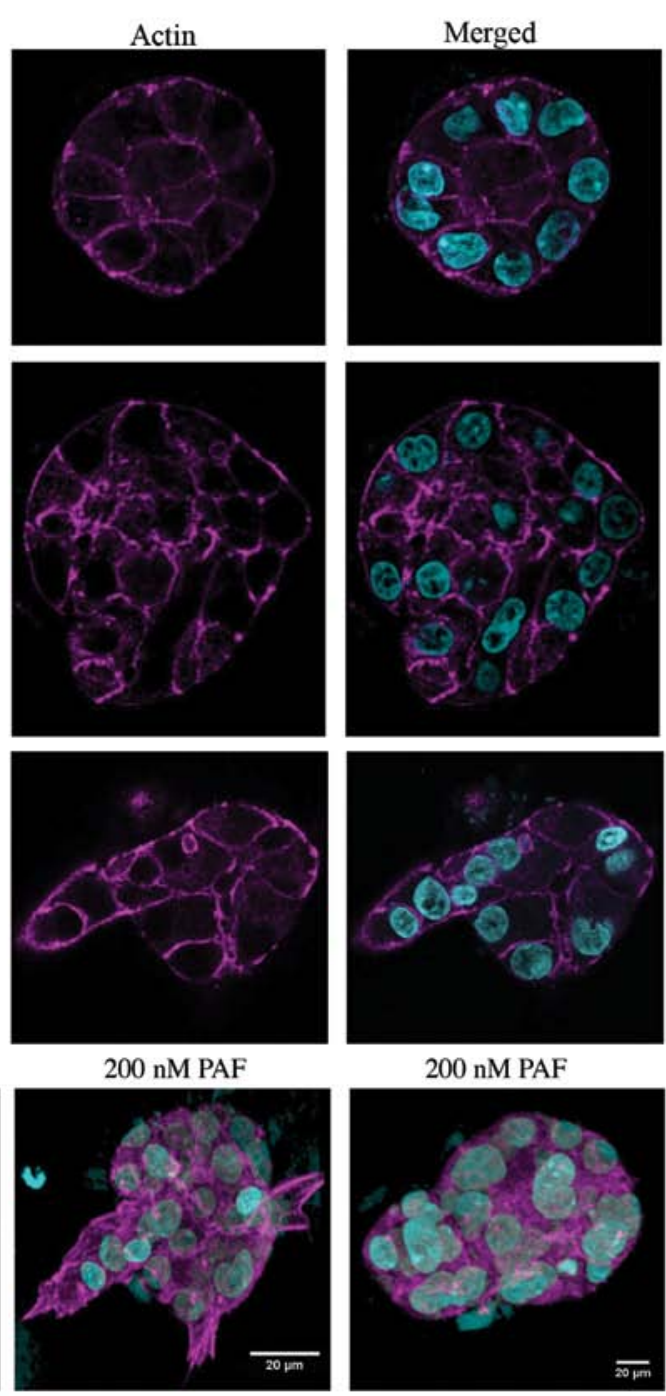

$200 \mathrm{nM}$ PAF
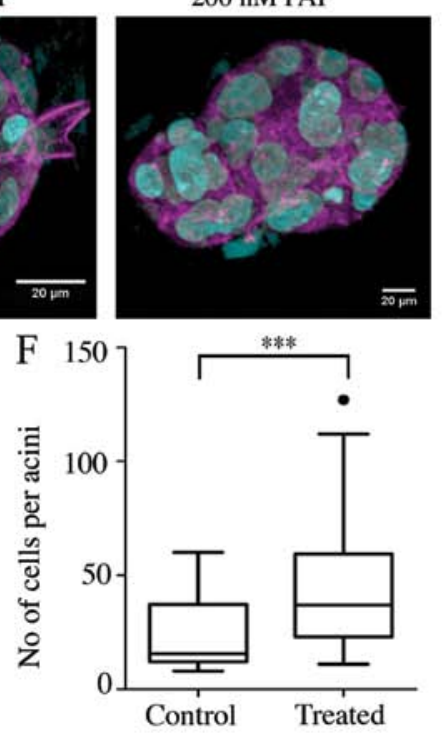
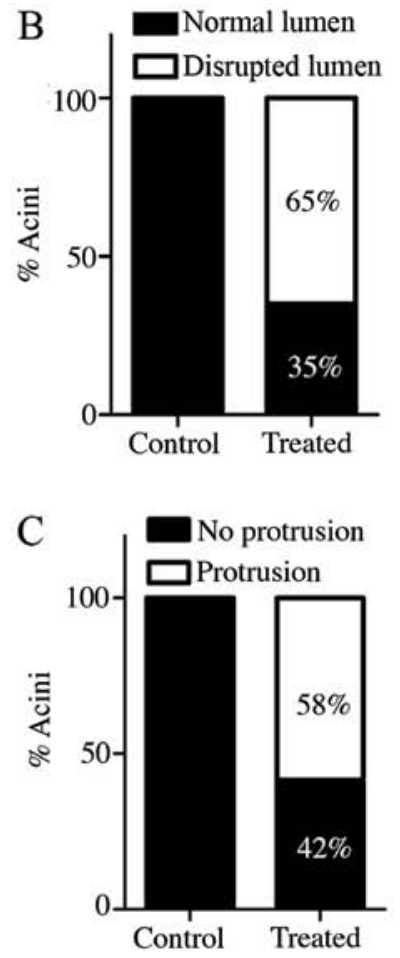

Figure 1. PAF induces formation of abnormal acinar structures in immortalized non-tumorigenic breast epithelial cell line MCF10A grown as 3D on-top cultures. (A) Representative images of day 20 MCF10A acini (centre optical section, $0.35 \mu$ m thickness). Top panel: untreated acini showing presence of distinct hollow lumen. Middle panel: acini treated with $200 \mathrm{nM}$ PAF on day 4, 8, 12 and 16; showing multiple layers of cells encircling a not so distinct lumen. Bottom panel: spheroids with protrusion-like structures extending out of acini. Graphs represent data quantified based on visual observation of 3D structures for (B) normal or disrupted lumen and (C) presence of protrusion like or 'bulb'-like structures. Data represent the pooled results from more than 3 independent experiments; $n=94$ acini. (D) 3D re-construction of the Z-optical sections to aid in visualization of the protrusions in acini following PAF treatment. (E and F) Box plots representing number of cell/acini and volume per acini respectively, quantified using Image-Pro Plus software (Media Cybernetics, USA). Mann-Whitney test was used to test the statistical significance, ${ }^{* * *} \mathrm{P}<0.0001$. Number of acini analyzed; $\mathrm{n}>30$.

motility of MDA-MB 231 cells at the single cell level time-lapse microscopy of sparsely seeded MDA-MB 231 cells on fibronectin coated chamber cover glass was performed for $4 \mathrm{~h}$. Tracking of cells was done and trajectory plots were obtained for the following cell treatments: untreated, PAF stimulated, WEB 2086 pre-treated followed by PAF stimulation and only WEB 2086 treated as shown in Fig. 4B. Further analysis of the cells revealed an increase in motility of cells upon PAF induction. There was a significant increase in the distance covered by the PAF-treated cells when compared to untreated cells as well as to cells that were pre-treated with WEB 2086 followed by PAF-stimulation in the time and conditions used in the study (Fig. 5A). Other parameters such as displacement (Fig. 5B) and velocity (Fig. 5C) were 

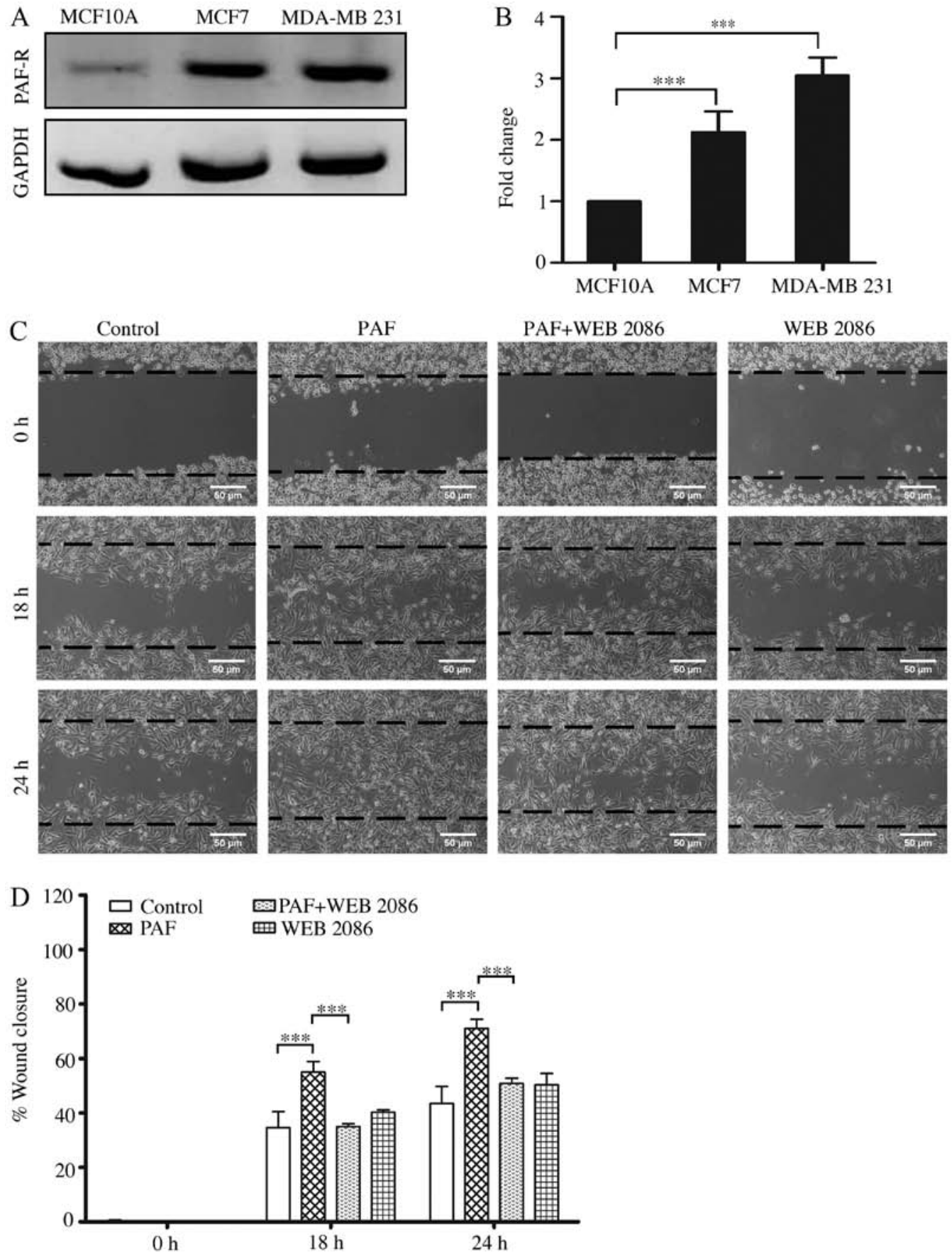

Figure 2. PAF increases collective cell migration in MDA-MB 231 cells. (A) RT-PCR analysis of PAF-receptor expression in non-transformed breast epithelial cell line MCF10A and breast cancer cell lines MCF-7 and MDA-MB 231. (B) Graphs represent mean \pm SEM fold change of expression level normalized to MCF10A PAF-R expression. Two-fold difference was considered to be significant. (C) Wound-healing assay demonstrating collective migration of MDA-MB 231 cells with or without treatment of $200 \mathrm{nM}$ PAF and/or $200 \mu \mathrm{M}$ WEB 2086 (PAF receptor antagonist). Scale bar: $50 \mu \mathrm{m}$. (D) Quantitative results for the data shown in (C). Percentage wound closures were calculated. Data represent mean \pm SEM ( $n=3$ independent experiments). Student's t-test was used to test the statistical significance of data, ${ }^{* * *} \mathrm{P}<0.001$.

observed to increase significantly following PAF treatment in comparison to untreated or PAF-stimulated and WEB 2086 pre-treated cells. All these effects induced by PAF were reversed upon pretreatment of cells with WEB 2086. The directionality, which is the ratio of euclidean distance to accumulated distance, did not show a significant change (Fig. 5D). Thus, the above results signify that PAF is capable of inducing motility and promoting random non-directional movement of MDA-MB 231 cells.

\section{Discussion}

Progression of cancer occurs due to a complex combination of uncontrolled growth of transformed cells, evasion of apoptosis and invasion of cancer cells into nearby tissues, finally resulting in metastasis and secondary tumor formation (24). Uncontrolled cell division coupled with evasion of apoptosis and migration (as single cells or clusters) can thus be considered as some of the key features of cancer cells. 

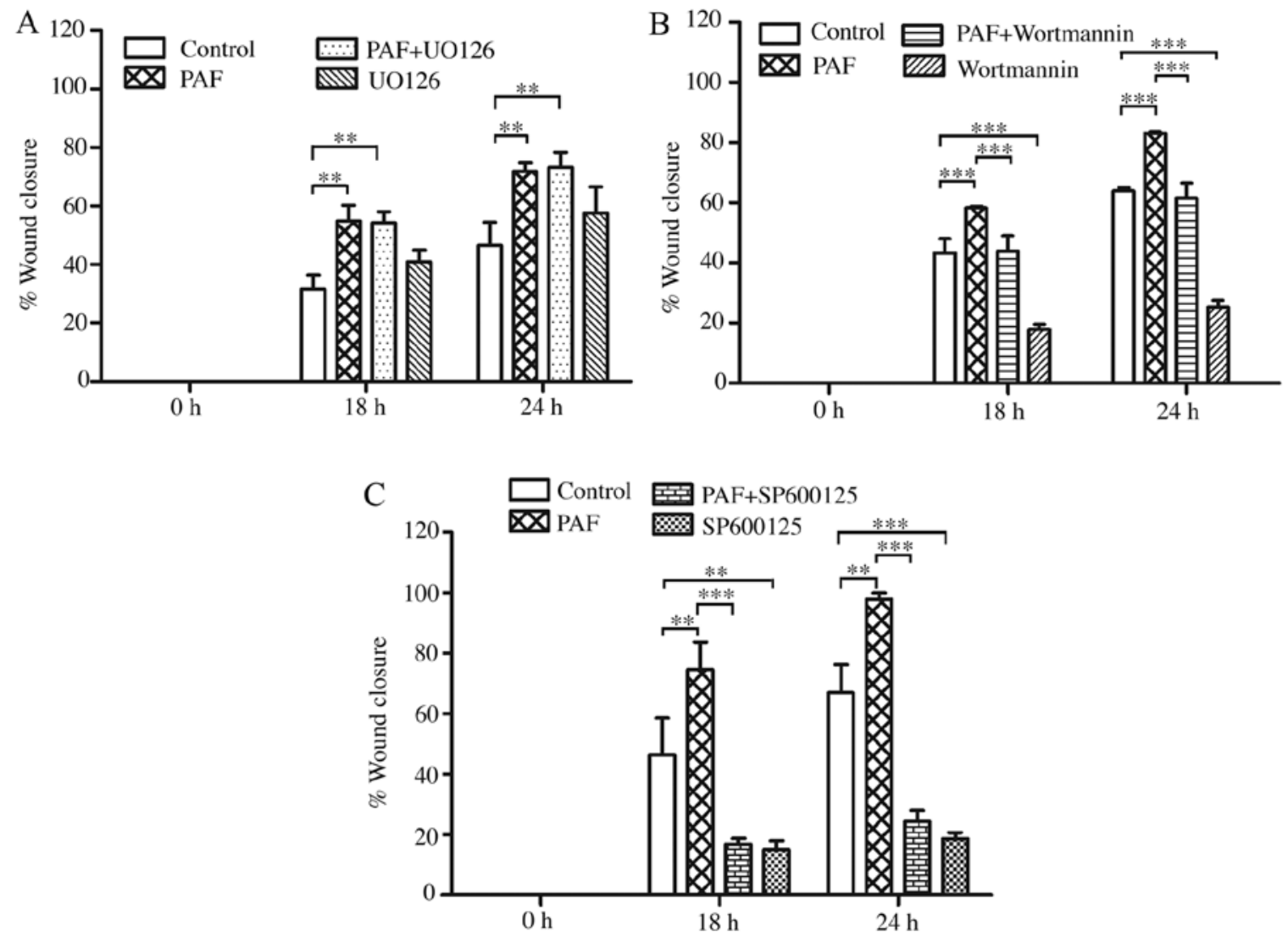

Figure 3. PAF induced migration is mediated via PI3-kinase pathway and/or JNK pathway but not via MAP-kinase pathway. Wound-healing assay of MDA-MB 231 cells with or without treatment of (A) PAF (200 nM) and/or $10 \mu \mathrm{M}$ UO126 (MEK inhibitor). (B) PAF (200 nM) and/or $200 \mathrm{nM}$ wortmannin (PI3-K inhibitor). (C) PAF (200 nM) and/or $75 \mu \mathrm{M}$ SP600125 (JNK inhibitor). Wound closure was measured at 0, 18 and $24 \mathrm{~h}$ for all treatments. Data represent mean \pm SEM ( $\mathrm{n}=3$ independent experiments). Wound area was measured using ImageJ software. Statistical significance was analyzed using Student's t-test, $\mathrm{P}<0.05$ is considered to be significant; ${ }^{* * *} \mathrm{P}<0.01,{ }^{* * * *} \mathrm{P}<0.001$.

Migration of cancer cells can be considered at two levels, namely, single cell migration and collective migration. Some of the carcinomas are known to progress through epithelial to mesenchymal transition, wherein the cells lose their cellcell adhesion and disseminate independently as single cells and finally lodge into a distant organ $(35,36)$. Besides single cell migration, recently, collective cell migration has also been implicated as the predominant mode of cancer cell invasion and metastasis (37). This process has been exhibited by most of the epithelial cancers including breast cancer, colorectal carcinoma, rhabdomyosarcoma, melanoma and oral squamous cell carcinoma (38-40). Histopathological studies of epithelial cancers in tumor regions, suggest the presence of clusters, sheets or chains of secondary cancer cells in the stroma surrounding the primary tumor (41). In addition to this, draining lymphatics were also found to contain clusters of metastasizing tumor cells, implying that small groups of tumor cells are capable of invading vasculatures and lymphatics in individuals with cancer (42). Thus migration, either collective or single cell is a vital phenomenon in the multi-step process of invasion and metastasis.

PAF and PAF-like lipids are present across various cell types and tissues such as neutrophils, macrophages and endothelial cells (43), as well as breast cancer tissues (11). One of the major sources of these lipids is chronic inflammatory microenvironments as well as the cancer cells per se $(3-5,16)$.
Different types of cells have been found to respond to such chemical stimuli, present in the microenvironment, in several ways such as proliferation, apoptosis, migration, to name a few. To investigate the role of PAF in early transformation, we used 3-dimensional cultures of MCF10A cells, which are non-transformed breast epithelial cells. 3D cultures of cells have been found to recapitulate the in vivo scenario largely. This model involves growing of cells on various extracellular matrix, one of the widely used matrix being Matrigel ${ }^{\circledR}$ which is derived from EHS tumors (23). This model aids in the easy identification of a transformed cell from a non-tumorigenic cell (21). The normal breast epithelial cells form growth-arrested multicellular acinar-like structures with a hollow lumen which closely resemble the epithelial cells lining the duct in the mammary tissue in vivo (34). In case of cancer cells, this well formed structure is disrupted leading to the formation of spheroids which are devoid of or have irregular lumen, multiple lumens or clusters of cells (44). The morphological features are a clear indication of the tumorigenic status of the cell lines. In our experiment MCF10A cells were under continuous exposure to $200 \mathrm{nM}$ PAF. While investigating the presence of a transformed phenotype, we observed $65 \%$ of the acini showing protrusion-like or 'bud like' phenotype. Such phenotypes have been related to collective migratory behavior of transformed cells $(44,45)$. Of the total acini imaged, 58\% showed disrupted lumen, which may imply that the cells in 
A
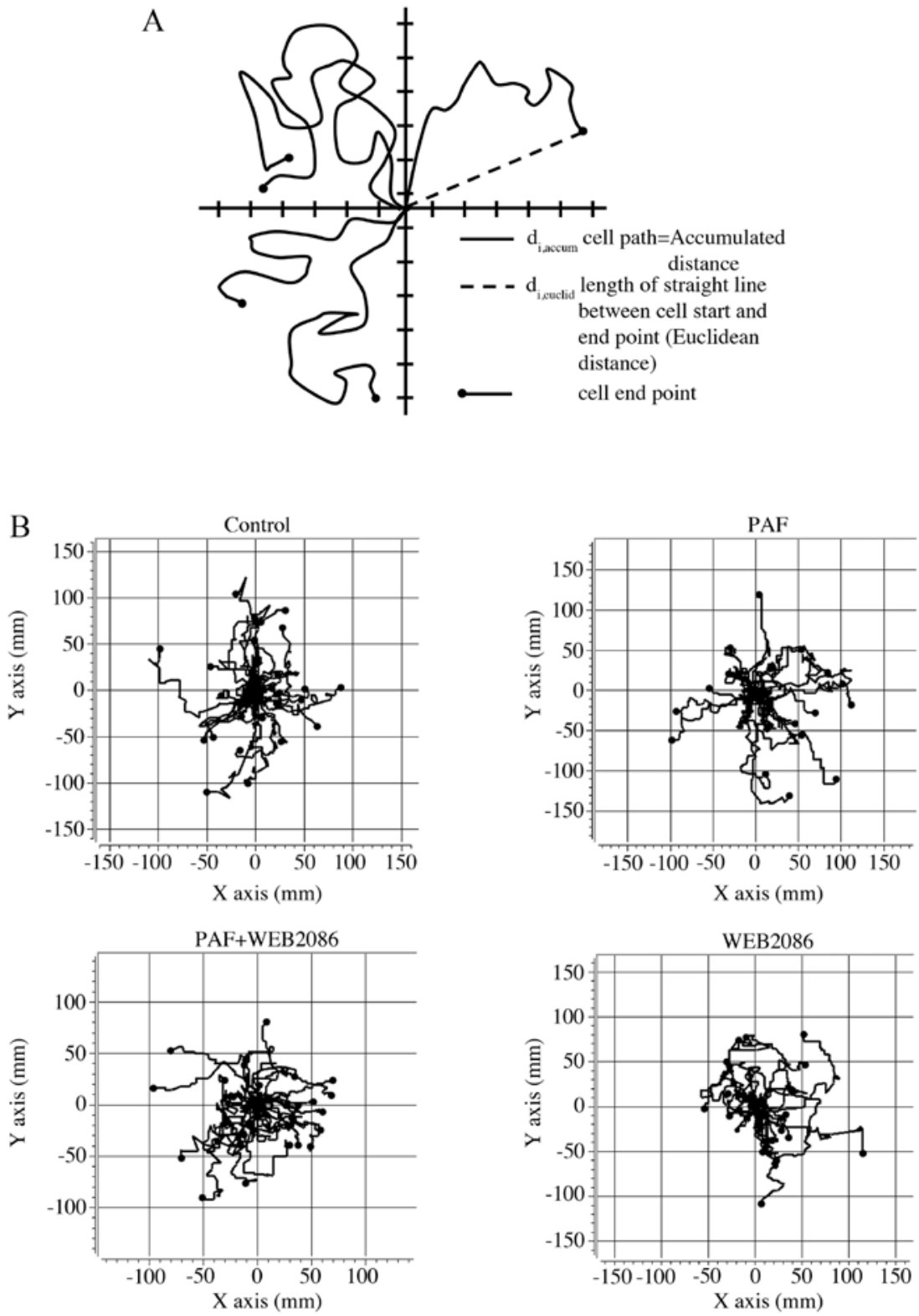

Figure 4. PAF induces chemokinesis at the single cell level in MDA-MB 231 cells. Time-lapse imaging of MDA-MB 231 cells seeded as sparse populations on fibronectin-coated dishes, with or without $200 \mathrm{nM} \mathrm{PAF}$ and/or $200 \mu \mathrm{M}$ WEB 2086 was performed for a period of $4 \mathrm{~h}$ with images taken at 2-min intervals Cells were tracked using ImageJ manual tracking plugin and analyzed using Chemotaxis and Migration tool (Ibidi GmbH, Munich, Germany) to calculate different motility parameters. (A) Diagrammatic representation of a trajectory plot depicting the calculation of the various chemokinetic parameters adapted from Ibidi chemotaxis and migration tool user manual. (B) Trajectory plots of various treatment combinations obtained using chemotaxis and migration tool.

the lumen may restrain apoptosis, which is another hallmark of epithelial cancers $(21,44,46)$. Apart from this, there was a significant increase in the number of cells per transformed acini, which may indicate the induction of proliferation or 'escape from the proliferative arrest', one of the hallmarks of cancer $(44,47)$. This is supported by published reports wherein PAF has been shown to induce proliferation in mouse vascular smooth muscle cells (48) as well as breast adenocarcinoma cells in tissue culture dishes (16). There have been contradictory reports to the same suggesting that the effect of PAF varies according to the type of cells and type of cancer (49). However, our results demonstrate the potential of PAF to induce proliferation in MCF10A cells under continuous exposure to PAF. These results appeal for further intervention with respect to the changes at the molecular level, which have subsequently given rise to such a drastic change in the morphogenesis of the non-tumorigenic cells.

PAF has been demonstrated to induce migration in many cell types such as eosinophils (50), human endothelial cells (43), peripheral blood lymphocytes (51), using in vivo as well as in vitro models and assays. However, to our knowledge, only one report pertained to the role of PAF in motility of breast cancer cells wherein PAF was demonstrated to induce chemotaxis as well as chemokinesis in the cells (16). Our results are in agreement with the published report by Bussolati et al (16), where we show PAF-induced chemokinesis at the single cell level in breast cancer cells. As per the dose and conditions used in our assay, we observed that PAF stimulated cells moved at a significantly increased velocity as well as traversed a larger distance as compared to untreated cells. Displacement was 

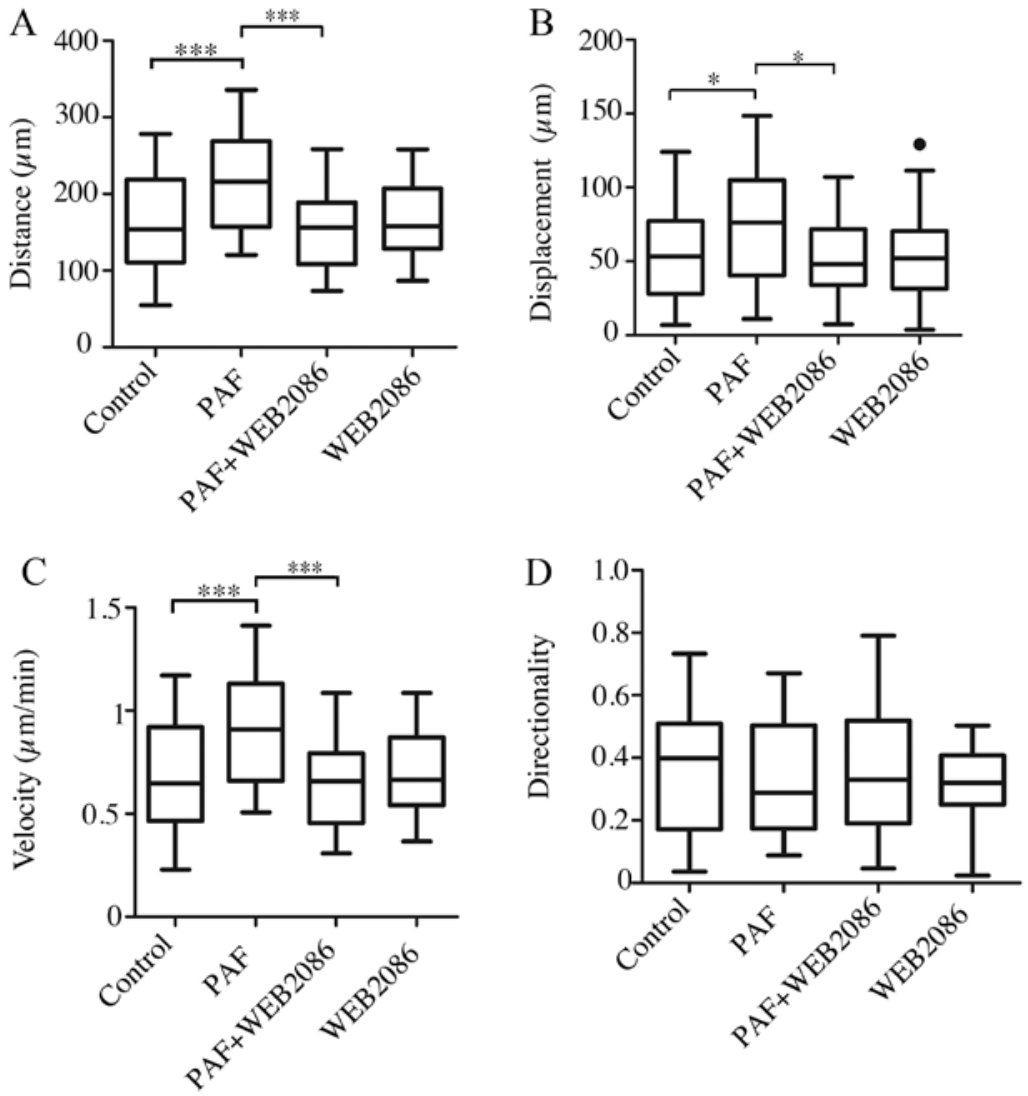

Figure 5. PAF induces chemokinesis without change in directionality at the single cell level in MDA-MB 231 cells. (A) Accumulated distance, (B) displacement, (C) velocity, (D) directionality. Box plots represent distribution of values calculated about the median. ( $\mathrm{n}=3$ independent experiments). Mann-Whitney test was used to test the statistical significance of data. ${ }^{*} \mathrm{P}<0.05,{ }^{* * *} \mathrm{P}<0.01,{ }^{* * * *} \mathrm{P}<0.001$

also significantly increased. However, there was no significant difference in the directionality indicating that PAF stimulation enhanced the random movement of the cells without inducing directionality. In order to investigate the role of PAF in collective cell migration, wound-healing assay, a traditional method to study the dynamic process of collective cell migration was performed (25). Wound-healing assay results implicated that PAF is capable of inducing collective cell migration in invasive MDA-MB 231 breast cancer cells. Pretreatment of cells with WEB 2086, a PAF receptor antagonist resulted in a significant decrease in PAF-stimulated motility of cells. Thus further substantiating the crucial role played by PAF in collective cell migration. Collectively these results imply that PAF can enhance cell migration and hence possibly promote metastasis in vivo.

To dissect out the motility pathway(s) that are involved in signaling in the highly metastatic MDA-MB 231 cells upon PAF induction, wound-healing assays were performed. PAF is known to act through PAF receptor and stimulate a number of signal transduction pathways and effectors such as PKCs, MAPKs, phospholipase C, paxillin, FAK, EGFR, and Src $(4,11,52)$. However, the downstream effectors of PAF and PAF-R, especially with respect to breast cancer progression and more precisely related to PAF-induced migration are not well elucidated. Metastasis is one of the areas of current interest in the field of cancer and search for better therapies related to it, still continues. Clarification on the downstream effectors of PAF-PAFR pathway involved in motility may, to some extent contribute to the designing of new strategies to treat this heterogeneous condition. To address this, MDA-MB 231 cells were subjected to treatment with inhibitors of well-known motility related pathways (53-55). PAF was found to induce collective cell migration in cells that were independent of the MAPK pathway but dependent on PI3-K as well as JNK pathways. However, inhibition of c-Jun reduced PAF motility almost completely while PI3-K inhibition resulted in partial inhibition of motility. Thus, these results indicate a possible role of PI3-K and/or JNK pathway in PAF-induced motility. However, this demands further investigation to elucidate the exact signal transduction pathway of PAF-induced motility.

In conclusion, role of PAF in cancer progression has been studied in the past (56). However, many questions pertaining to its role in breast cancer are yet to be answered. In this study, we have demonstrated the possible role of PAF in inducing transformation of non-tumorigenic breast epithelial cells grown as spheroids as well as promoting migration of metastatic breast cancer cells. Nonetheless, this study calls for further detailed investigation to unravel some of the interesting questions raised with respect to the involvement of signaling pathways in PAF-induced motility.

\section{Acknowledgements}

We thank Professor L.S. Shashidhara for critical reading of the manuscript and providing useful suggestions, Lahiri lab members for helpful comments and discussions, Drs Aurnab 
Ghose and Nagaraj Balasubramanian for their suggestions with the single cell migration assay, Vijay Vittal at the IISER, Pune Microscopy facility for his help with microscopy and Dr Kundan Sengupta for sharing with us the Image-Pro Plus software (Media Cybernetics, USA). This study is supported by Indian Institute of Science Education and Research, Pune Core funding (BT/PR8699/MED/30/1018/2013). L.A.V. is funded through DST-INSPIRE fellowship while A.K.A. and N.K. are funded through DST-INSPIRE scholarship.

\section{References}

1. Ramos-Nino ME: The role of chronic inflammation in obesity-associated cancers. ISRN Oncol 2013: 697521, 2013.

2. Sounni NE and Noel A: Targeting the tumor microenvironment for cancer therapy. Clin Chem 59: 85-93, 2013.

3. BussolinoF,Arese M, Montrucchio G,Barra L,PrimoL, BenelliR, Sanavio F, Aglietta M, Ghigo D and Rola-Pleszczynski MR: Platelet activating factor produced in vitro by Kaposi's sarcoma cells induces and sustains in vivo angiogenesis. J Clin Invest 96 940-952, 1995.

4. Caiazza F, Harvey BJ and Thomas W: Cytosolic phospholipase A2 activation correlates with HER2 overexpression and mediates estrogen-dependent breast cancer cell growth. Mol Endocrinol 24: 953-968, 2010.

5. Camussi G, Montrucchio G, Lupia E, Arese M and Bussolino F: Platelet-activating factor and angiogenesis. In: Platelet-Activating Factor and Related Lipid Mediators 2. Nigam S, Kunkel G and Prescott S (eds). Vol. 416, Springer US, pp231-234, 1996.

6. Zhu T, Gobeil F, Vazquez-Tello A, Leduc M, Rihakova L, Bossolasco M, Bkaily G, Peri K, Varma DR, Orvoine R, et al: Intracrine signaling through lipid mediators and their cognate nuclear G-protein-coupled receptors: A paradigm based on PGE2, PAF, and LPA1 receptors. Can J Physiol Pharmacol 84 377-391, 2006

7. Stafforini DM, McIntyre TM, Zimmerman GA and Prescott SM: Platelet-activating factor, a pleiotrophic mediator of physiological and pathological processes. Crit Rev Clin Lab Sci 40: 643-672, 2003.

8. Prescott SM, Zimmerman GA, Stafforini DM and McIntyre TM: Platelet-activating factor and related lipid mediators. Annu Rev Biochem 69: 419-445, 2000.

9. Chao W and Olson MS: Platelet-activating factor: Receptors and signal transduction. Biochem J 292: 617-629, 1993.

10. Ryan SD, Harris CS, Mo F, Lee H, Hou ST, Bazan NG, Haddad PS ArnasonJT and Bennett SA: Platelet activating factor-induced neuronal apoptosis is initiated independently of its G-protein coupled PAF receptor and is inhibited by the benzoate orsellinic acid. J Neurochem 103: 88-97, 2007.

11. McHowat J, Gullickson G, Hoover RG, Sharma J, Turk J and Kornbluth J: Platelet-activating factor and metastasis: Calcium-independent phospholipase A2 $\beta$ deficiency protects against breast cancer metastasis to the lung. Am J Physiol Cell Physiol 300: C825-C832, 2011.

12. Melnikova V and Bar-Eli M: Inflammation and melanoma growth and metastasis: The role of platelet-activating factor (PAF) and its receptor. Cancer Metastasis Rev 26: 359-371, 2007.

13. Im SY, Ko HM, Kim JW, Lee HK, Ha TY, Lee HB, Oh SJ, Bai S, Chung KC, Lee YB, et al: Augmentation of tumor metastasis by platelet-activating factor. Cancer Res 56: 2662-2665, 1996.

14. Melnikova VO, Villares GJ and Bar-Eli M: Emerging roles of PAR-1 and PAFR in melanoma metastasis. Cancer Microenviron 1: 103-111, 2008.

15. Zhang L, Wang D, Jiang W, Edwards D, Qiu W, Barroilhet LM, Rho JH,Jin L, Seethappan V, Vitonis A, et al: Activated networking of platelet activating factor receptor and FAK/STAT1 induces malignant potential in BRCA1-mutant at-risk ovarian epithelium. Reprod Biol Endocrinol 8: 74, 2010.

16. Bussolati B, Biancone L, Cassoni P, Russo S, Rola-PleszczynskiM, Montrucchio G and Camussi G: PAF produced by human breast cancer cells promotes migration and proliferation of tumor cells and neo-angiogenesis. Am J Pathol 157: 1713-1725, 2000.

17. Kravchenko VV, Pan Z, Han J, Herbert JM, Ulevitch RJ and Ye RD: Platelet-activating factor induces NF-kappa B activation through a G protein-coupled pathway. J Biol Chem 270: 14928-14934, 1995.
18. Ishii S, Nagase T, Tashiro F, Ikuta K, Sato S, Waga I, Kume K, Miyazaki J and Shimizu T: Bronchial hyperreactivity, increased endotoxin lethality and melanocytic tumorigenesis in transgenic mice overexpressing platelet-activating factor receptor. EMBO J 16: 133-142, 1997.

19. Cellai C, Laurenzana A, Vannucchi AM, Caporale R, Paglierani M, Di Lollo S, Pancrazzi A and Paoletti F: Growth inhibition and differentiation of human breast cancer cells by the PAFR antagonist WEB-2086. Br J Cancer 94: 1637-1642, 2006.

20. Robert EG and Hunt JD: Lipid messengers as targets for antiangiogenic therapy. Curr Pharm Des 7: 1615-1626, 2001.

21. Debnath J, Muthuswamy SK and Brugge JS: Morphogenesis and oncogenesis of MCF-10A mammary epithelial acini grown in three-dimensional basement membrane cultures. Methods 30: 256-268, 2003

22. Petersen OW, Rønnov-Jessen L, Howlett AR and Bissell MJ: Interaction with basement membrane serves to rapidly distinguish growth and differentiation pattern of normal and malignant human breast epithelial cells. Proc Natl Acad Sci USA 89: 9064-9068, 1992.

23. Bissell MJ and Radisky D: Putting tumours in context. Nat Rev Cancer 1: 46-54, 2001

24. Deisboeck TS and Couzin ID: Collective behavior in cancer cell populations. Bioessays 31: 190-197, 2009.

25. Riahi R, Yang Y, Zhang DD and Wong PK: Advances in wound-healing assays for probing collective cell migration. J Lab Autom 17: 59-65, 2012.

26. Kuroda Y and Furuyama J: Physiological and biochemical studies of effects of mitomycin C on strain HeLa cells in cell culture. Cancer Res 23: 682-687, 1963.

27. Casals-Stenzel J, Muacevic G and Weber KH: Pharmacological actions of WEB 2086, a new specific antagonist of platelet activating factor. J Pharmacol Exp Ther 241: 974-981, 1987.

28. Cellai C, Laurenzana A, Vannucchi AM, Della Malva N Bianchi L and Paoletti F: Specific PAF antagonist WEB-2086 induces terminal differentiation of murine and human leukemia cells. FASEB J 16: 733-735, 2002.

29. Du J, Sun C, Hu Z, Yang Y, Zhu Y, Zheng D, Gu L and Lu X: Lysophosphatidic acid induces MDA-MB-231 breast cancer cells migration through activation of PI3K/PAK1/ERK signaling. PLoS One 5: e15940, 2010.

30. Powis G, Bonjouklian R, Berggren MM, Gallegos A, Abraham R, Ashendel C, Zalkow L, Matter WF, Dodge J, Grindey G, et al: Wortmannin, a potent and selective inhibitor of phosphatidylinositol-3-kinase. Cancer Res 54: 2419-2423, 1994.

31. Bennett BL, Sasaki DT, Murray BW, O'Leary EC, Sakata ST, Xu W, Leisten JC, Motiwala A, Pierce S, Satoh Y, et al: SP600125, an anthrapyrazolone inhibitor of Jun N-terminal kinase. Proc Natl Acad Sci USA 98: 13681-13686, 2001.

32. Bodakuntla S, Libi AV, Sural S, Trivedi P and Lahiri M: N-nitroso-N-ethylurea activates DNA damage surveillance pathways and induces transformation in mammalian cells. BMC Cancer 14: $287,2014$.

33. Lee GY, Kenny PA, Lee EH and Bissell MJ: Three-dimensional culture models of normal and malignant breast epithelial cells. Nat Methods 4: 359-365, 2007.

34. Vidi PA, Bissell MJ and Lelièvre SA: Three-dimensional culture of human breast epithelial cells: The how and the why. Methods Mol Biol 945: 193-219, 2013.

35. Sleeman JP and Thiery JP: SnapShot: The epithelial-mesenchymal transition. Cell 145: 162.e1, 2011.

36. Thiery JP, Acloque H, Huang RY and Nieto MA: Epithelial-mesenchymal transitions in development and disease. Cell 139: 871-890, 2009

37. Bidard FC, Pierga JY, Vincent-Salomon A and Poupon MF: A 'class action' against the microenvironment: Do cancer cells cooperate in metastasis? Cancer Metastasis Rev 27: 5-10, 2008.

38. Friedl P, Noble PB, Walton PA, Laird DW, Chauvin PJ, Tabah RJ, Black M and Zänker KS: Migration of coordinated cell clusters in mesenchymal and epithelial cancer explants in vitro. Cancer Res 55: 4557-4560, 1995.

39. Hegerfeldt Y, Tusch M, Bröcker EB and Friedl P: Collective cell movement in primary melanoma explants: Plasticity of cell-cell interaction, beta1-integrin function, and migration strategies. Cancer Res 62: 2125-2130, 2002.

40. Nabeshima K, Inoue T, Shimao Y, Kataoka $H$ and Koono M: Cohort migration of carcinoma cells: Differentiated colorectal carcinoma cells move as coherent cell clusters or sheets. Histol Histopathol 14: 1183-1197, 1999. 
41. Friedl P and Gilmour D: Collective cell migration in morphogenesis, regeneration and cancer. Nat Rev Mol Cell Biol 10: 445-457, 2009.

42. Rørth P: Collective cell migration. Annu Rev Cell Dev Biol 25: 407-429, 2009.

43. Montrucchio G, Lupia E, Battaglia E, Del Sorbo L, Boccellino M, Biancone L, Emanuelli G and Camussi G: Platelet-activating factor enhances vascular endothelial growth factor-induced endothelial cell motility and neoangiogenesis in a murine matrigel model. Arterioscler Thromb Vasc Biol 20: 80-88, 2000.

44. Debnath J and Brugge JS: Modelling glandular epithelial cancers in three-dimensional cultures. Nat Rev Cancer 5: 675-688, 2005.

45. Friedl P, Hegerfeldt $\mathrm{Y}$ and Tusch M: Collective cell migration in morphogenesis and cancer. Int J Dev Biol 48: 441-449, 2004.

46. Debnath J, Mills KR, Collins NL, Reginato MJ, Muthuswamy SK and Brugge JS: The role of apoptosis in creating and maintaining luminal space within normal and oncogene-expressing mammary acini. Cell 111: 29-40, 2002.

47. Hanahan D and Weinberg RA: Hallmarks of cancer: The next generation. Cell 144: 646-674, 2011.

48. Gaumond F, Fortin D, Stankova J and Rola-Pleszczynski M: Differential signaling pathways in platelet-activating factor-induced proliferation and interleukin- 6 production by rat vascular smooth muscle cells. J Cardiovasc Pharmacol 30: 169-175, 1997.

49. Wang $\mathrm{H}$ and Chakrabarty S: Platelet-activating factor activates mitogen-activated protein kinases, inhibits proliferation, induces differentiation and suppresses the malignant phenotype of human colon carcinoma cells. Oncogene 22: 2186-2191, 2003.
50. Schweizer RC, van Kessel-Welmers BA, Warringa RA, Maikoe T, Raaijmakers JA, Lammers JW and Koenderman L: Mechanisms involved in eosinophil migration. Platelet-activating factor-induced chemotaxis and interleukin-5-induced chemokinesis are mediated by different signals. J Leukoc Biol 59: 347-356, 1996.

51. McFadden RG, Bishop MA, Caveney AN and Fraher LJ: Effect of platelet activating factor (PAF) on the migration of human lymphocytes. Thorax 50: 265-269, 1995.

52. Aponte M, Jiang W, Lakkis M, Li MJ, Edwards D, Albitar L, Vitonis A, Mok SC, Cramer DW and Ye B: Activation of platelet-activating factor receptor and pleiotropic effects on tyrosine phospho-EGFR/Src/FAK/paxillin in ovarian cancer. Cancer Res 68: 5839-5848, 2008.

53. Huang C, Rajfur Z, Borchers C, Schaller MD and Jacobson K: JNK phosphorylates paxillin and regulates cell migration. Nature 424: 219-223, 2003.

54. Lopez-Ilasaca M: Signaling from G-protein-coupled receptors to mitogen-activated protein (MAP)-kinase cascades. Biochem Pharmacol 56: 269-277, 1998.

55. Wymann MP and Pirola L: Structure and function of phosphoinositide 3-kinases. Biochim Biophys Acta 1436: 127-150, 1998.

56. Tsoupras AB, Iatrou $C$, Frangia $C$ and Demopoulos $C A$ : The implication of platelet activating factor in cancer growth and metastasis: Potent beneficial role of PAF-inhibitors and antioxidants. Infect Disord Drug Targets 9: 390-399, 2009. 